Article

\title{
Regional Differences in COVID-19 Vaccine Hesitancy in December 2020: A Natural Experiment in the French Working-Age Population
}

\author{
Fanny Velardo ${ }^{1,2}$, Verity Watson ${ }^{3}$, Pierre Arwidson ${ }^{4}$, François Alla ${ }^{1,2}{ }^{\oplus}$, Stéphane Luchini ${ }^{5}$, \\ Michaël Schwarzinger ${ }^{1,2, *}$ and CoVaMax Study Group ${ }^{\dagger}$ \\ 1 Department of Methodology and Innovation in Prevention, \\ Bordeaux University Hospital, 33000 Bordeaux, France; fanny.velardo@chu-bordeaux.fr (F.V.); \\ francois.alla@u-bordeaux.fr (F.A.) \\ 2 University of Bordeaux, Inserm UMR 1219-Bordeaux Population Health, 33000 Bordeaux, France \\ 3 Health Economics Research Unit (HERU), University of Aberdeen, Aberdeen AB25 2ZD, UK; \\ v.watson@abdn.ac.uk \\ 4 Santé Publique France, 94410 Saint Maurice, France; pierre.arwidson@santepubliquefrance.fr \\ 5 CNRS, EHESS, Centrale Marseille, AMSE, Aix-Marseille University, 13001 Marseille, France; \\ stephane.luchini@univ-amu.fr \\ * Correspondence: michael.schwarzinger@chu-bordeaux.fr \\ + Members listed at end of paper.
}

\section{check for} updates

Citation: Velardo, F.; Watson, V.; Arwidson, P.; Alla, F.; Luchini, S.; Schwarzinger, M.; CoVaMax Study Group. Regional Differences in COVID-19 Vaccine Hesitancy in December 2020: A Natural Experiment in the French Working-Age Population. Vaccines 2021, 9, 1364. https://doi.org/ $10.3390 /$ vaccines 9111364

Academic Editor: S. Louise Cosby

Received: 3 August 2021

Accepted: 17 November 2021

Published: 20 November 2021

Publisher's Note: MDPI stays neutral with regard to jurisdictional claims in published maps and institutional affiliations.

Copyright: (c) 2021 by the authors. Licensee MDPI, Basel, Switzerland. This article is an open access article distributed under the terms and conditions of the Creative Commons Attribution (CC BY) license (https:/ / creativecommons.org/licenses/by/ $4.0 /)$.

\begin{abstract}
It can be assumed that higher SARS-CoV-2 infection risk is associated with higher COVID19 vaccination intentions, although evidence is scarce. In this large and representative survey of 6007 adults aged 18-64 years and residing in France, 8.1\% (95\% CI, 7.5-8.8) reported a prior SARSCoV-2 infection in December 2020, with regional variations according to an East-West gradient $(p<0.0001)$. In participants without prior SARS-CoV-2 infection, COVID-19 vaccine hesitancy was substantial, including $41.3 \%$ (95\% CI, 39.8-42.8) outright refusal of COVID-19 vaccination. Taking into account five characteristics of the first approved vaccines (efficacy, duration of immunity, safety, country of the vaccine manufacturer, and place of administration) as well as the initial setting of the mass vaccination campaign in France, COVID-19 vaccine acceptance would reach 43.6\% (95\% CI, 43.0-44.1) at best among working-age adults without prior SARS-CoV-2 infection. COVID-19 vaccine acceptance was primarily driven by vaccine characteristics, sociodemographic and attitudinal factors. Considering the region of residency as a proxy of the likelihood of getting infected, our study findings do not support the assumption that SARS-CoV-2 infection risk is associated with COVID-19 vaccine acceptance.
\end{abstract}

Keywords: SARS-CoV-2; COVID-19; mass vaccination; anti-vaccination behavior; vaccine hesitancy; survey experiment; discrete choice experiment; France

\section{Introduction}

On 11 March 2020, the World Health Organization declared the severe acute respiratory syndrome coronavirus 2 (SARS-CoV-2) outbreak in China to be a pandemic [1]. All European countries implemented "stop and go" strategies with more or less restrictive non-pharmaceutical interventions during 2020 to reduce SARS-CoV-2 transmission [2,3] and coronavirus disease-19 (COVID-19) mortality [4,5]. By mid-November 2020, COVID-19 vaccination had become a reality with the announcement of safe and effective candidate vaccines meeting all primary endpoints in large phase 3 trials [6-8]. Mass vaccination campaigns started in the European Union (EU) immediately after the approval of a first COVID-19 vaccine on 21 December 2020 [9]. However, beyond global constraints on vaccine supply for mass vaccination campaigns, COVID-19 vaccine hesitancy may represent 
a major hurdle to reaching herd immunity and controlling the SARS-CoV-2 pandemic without non-pharmaceutical interventions in each country [10-12].

Vaccine hesitancy is defined by a delay in acceptance or refusal of vaccination despite availability of vaccination services [13]. Vaccine hesitancy is multifactorial [14] and varies across countries and over time [15]. In this regard, COVID-19 vaccine hesitancy is no exception [16-18]. However, high-income countries were diversely affected by the SARS$\mathrm{CoV}-2$ pandemic $[4,5]$. It may be assumed that countries with higher prevalence rates of SARS-CoV-2 infection may experience higher COVID-19 vaccination intentions [19-21] and reach herd immunity at a faster rate due to the combined effect of higher natural immunity and vaccine uptake.

The large and rapid initial peak of SARS-CoV-2 infection in the North-East and Ilede-France regions of France [22] provides a unique natural experiment to understand the association between SARS-CoV-2 infection risk and COVID-19 vaccination intentions at the regional level. The main aim of this study was to assess this association in the French working-age population (18-64 years) who is at high risk of SARS-CoV-2 infection as compared to the elderly $[22,23]$. We conducted a survey experiment in December 2020 among a large and representative sample of twelve regions of France and found that COVID-19 vaccine hesitancy was substantial and primarily driven by vaccine characteristics, sociodemographic and attitudinal factors, but unrelated to SARS-CoV-2 infection risk.

\section{Materials and Methods}

\subsection{Study Design}

We conducted a cross-sectional survey among a representative sample of 6007 adults aged 18-64 years residing in twelve of thirteen regions of France (Auvergne-RhôneAlpes (ARA), Bourgogne-Franche-Comté (BFC), Bretagne (BRE), Centre-Val de Loire (CVL), Grand Est (GES), Hauts-de-France (HDF), Ile-de-France (IDF), Normandie (NOR), Nouvelle-Aquitaine (NAQ), Occitanie (OCC), Pays-de-la-Loire (PDL), and Provence-AlpesCôte-d'Azur (PAC)). The survey was fielded from 30 November to 16 December 2020, during the second wave of the SARS-CoV-2 pandemic and the regional extension of the curfew to the whole country [2]. Participants were selected for this survey from an online survey research panel, which was developed and is maintained by BVA (Paris, France) and consists of more than 700,000 French adults. Pre-existing information on the participants was used by BVA to determine eligibility and draw a stratified random sample, with oversampling of participants with low response rates. Sampling was stratified to match French official census statistics by region of residency (gender, age group, education level, household size, and area of residence). The survey was not conducted in Corsica, as this thirteenth region was underrepresented in the BVA online survey research panel.

Participants completed a self-reported online questionnaire eliciting background information, past vaccination behavior, risk factors of a severe form of COVID-19, COVID-19 experience, and risk perceptions about COVID-19 [24]. At the end of the online questionnaire, participants were randomized to two survey experiments assessing COVID-19 vaccine hesitancy: $20 \%$ completed the same survey experiment that was fielded in June 2020 [24], and the results of this replication study are reported elsewhere [25]; 80\% completed a new survey experiment, and the study results across French regions are reported in this paper (Figure 1). 


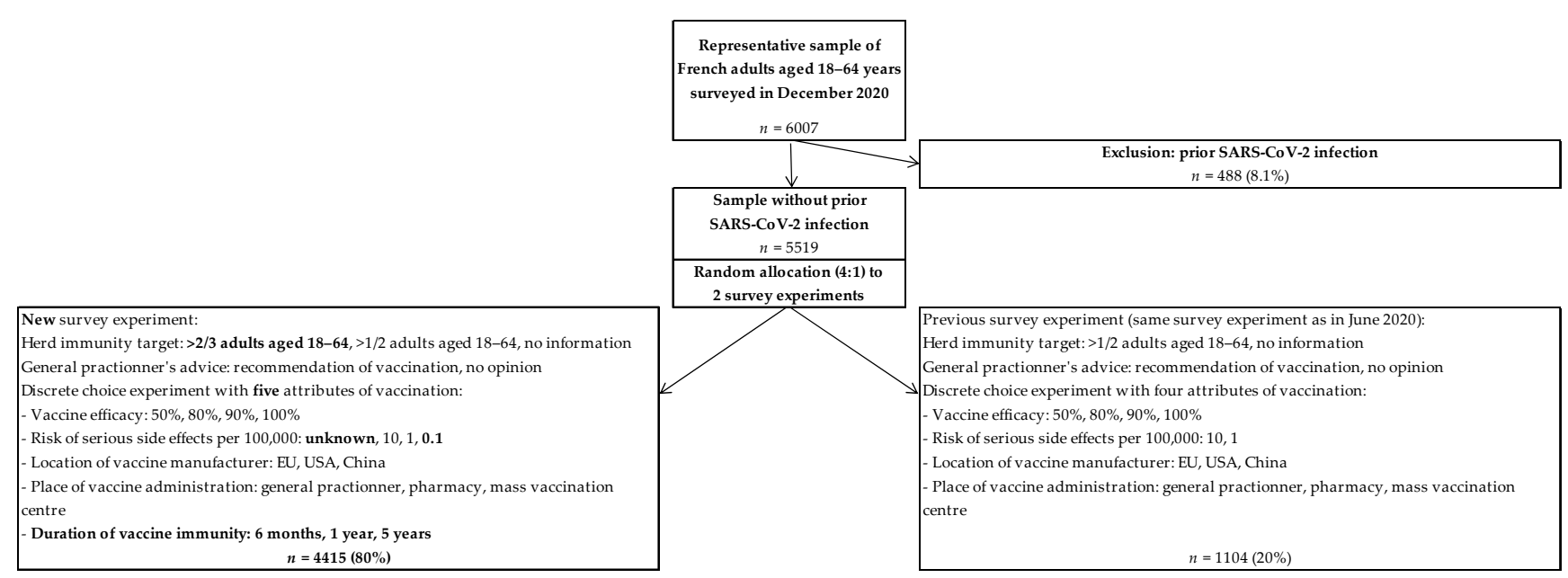

Figure 1. Study flowchart.

\subsection{New Survey Experiment Conducted in December 2020}

The survey experiment consisted of two sections [24]: background information on COVID-19 vaccination and the elicitation of vaccine acceptance based on vaccine characteristics. In the first section, all participants were provided with a full page presenting general information about COVID-19 vaccination. In addition, participants were randomized to two different information blocks according to a full factorial design. In block 1, participants were randomized to information about the collective benefits of herd immunity with a herd immunity target in adults aged 18-64 years ( $>2 / 3$ adults, $>1 / 2$ adults, or no information) [26]. In block 2, participants were randomized across the general practitioner's advice about COVID-19 vaccination (recommendation or no opinion) $[27,28]$.

In the second section, COVID-19 vaccine acceptance was elicited using a discrete choice experiment [29]. Participants completed a series of eight tasks. In each task, they chose between having one of two hypothetical vaccines or no vaccination. As compared to our previous survey experiment [24], the hypothetical vaccines differed on five rather than four characteristics (the duration of immunity conferred by vaccination was added) and four rather than two levels of vaccine safety. The five characteristics (levels) were vaccine efficacy to reduce COVID-19 risk (50\%, $80 \%, 90 \%$, or $100 \%)$; vaccine safety regarding the risk serious side-effects (unknown, 1/10,000,1/100,000, or 1/1,000,000 vaccinated people); country of the vaccine manufacturer (headquarters in EU, United States of America (USA), or China); place of administration (general practitioner practice, local pharmacy, or mass vaccination center); and duration of vaccine immunity (booster needed every 6 months, booster needed every year, or no booster needed).

Based on these vaccine characteristics and levels, there were 432 hypothetical vaccines and 93,096 possible choice tasks between two hypothetical vaccines. A D-efficient experimental design was created with NGENE software (ChoiceMetrics, Sydney, NSW, Australia, 2014) to reduce these to 16 choice tasks with 32 hypothetical vaccines, and participants were randomized to one of two blocks of eight task choices (Supplementary Table S1). A choice task example is provided in Figure 2. 


\begin{tabular}{|c|c|c|c|}
\hline Choice task & Vaccination scenario A & Vaccination scenario B & No vaccination \\
\hline $\begin{array}{l}\text { Risk of being infected } \\
\text { with SARS-CoV-2 }\end{array}$ & $\begin{array}{c}\text { The vaccine reduces your risk of } \\
\text { having COVID-19 disease by } \mathbf{1 0 0} \% \\
\text { and reduces the risk of people } \\
\text { around you getting infected }\end{array}$ & $\begin{array}{l}\text { The vaccine reduces your risk of } \\
\text { having COVID-19 disease by } \mathbf{8 0} \% \\
\text { and reduces the risk of people } \\
\text { around you getting infected }\end{array}$ & $\begin{array}{l}\text { Your risk of having COVID-19 } \\
\text { disease depends on the number of } \\
\text { cases in your area and the } \\
\text { protective measures you take on a } \\
\text { daily basis }\end{array}$ \\
\hline $\begin{array}{l}\text { Risk of rare but serious side } \\
\text { effects from the vaccine }\end{array}$ & $\begin{array}{c}\text { The risk is } \\
1 \text { per } \mathbf{1 0 0 , 0 0 0} \text { vaccinated people }\end{array}$ & $\begin{array}{c}\text { The risk is } \\
1 \text { per } \mathbf{1 0 , 0 0 0} \text { vaccinated people }\end{array}$ & You take no risk \\
\hline Manufacturer of the vaccine & $\begin{array}{l}\text { Headquarters in } \\
\text { European Union }\end{array}$ & $\begin{array}{c}\text { Headquarters in } \\
\text { United States of America }\end{array}$ & Not applicable \\
\hline Place to be vaccinated & At your general practice & At mass vaccination centre & Not applicable \\
\hline Duration of vaccine immunity & Vaccine booster every 6 months & Vaccine booster every year & Not applicable \\
\hline What would you choose? & $\begin{array}{l}\text { I would be vaccinated } \\
\text { in the scenario A }\end{array}$ & $\begin{array}{l}\text { I would be vaccinated } \\
\text { in the scenario } B\end{array}$ & $\square$ I would not be vaccinated \\
\hline
\end{tabular}

Figure 2. Choice task example in the discrete choice experiment used in December 2020.

\subsection{Statistical Analysis}

The proportion of participants who reported prior SARS-CoV-2 infection was estimated in the whole sample, overall and by region, with use of an exact $95 \%$ confidence interval $(95 \% \mathrm{CI})$ for a binomial proportion.

COVID-19 vaccine acceptance was estimated in the sample without prior SARS-CoV-2 infection with use of a single hurdle repeated discrete choice model $[24,30]$. Briefly, this behavioral model is a two-part model that specifies one decision process underlying outright vaccination refusal (i.e., serial non-vaccination in all eight choice tasks) and another decision process underlying vaccine acceptance (or conversely vaccine hesitancy) depending on vaccine characteristics. The probability of outright vaccination refusal and associated independent factors was analyzed using a logit regression model. The probability of vaccine acceptance based on vaccine characteristics and other independent factors was analyzed using a conditional logit regression model for repeated discrete choices in participants who did not refuse vaccination outright. Altogether, the two-part model provides an estimation of COVID-19 vaccine acceptance, i.e., $\left(1-p_{\text {outright vaccination refusal }}\right) \times p_{\text {vaccine acceptance. }}$

The same set of independent factors was used to explain both decision processes and included the following: region of residency, six stratification variables used in the sampling procedure by region (gender, age group, educational level, household size (number of adults and number of children), and area of residence); 15 variables related to vaccination behavior (working status including health-care worker status, unemployment or furlough because of the COVID-19 health crisis, compliance with recommended vaccinations in the past, vaccination against 2020/21 influenza, six risk factors for a severe form of COVID-19 (pregnancy, smoking status, obesity, hypertension, diabetes, and other chronic condition), four variables related to COVID-19 experience (COVID-19 symptoms, test for SARS-CoV-2 infection diagnosis, knowledge of people who got COVID-19, and curfew in the municipality), and perceived severity of COVID-19 if infected); and the randomized information blocks.

Probabilities and $95 \%$ CI of outright vaccination refusal and COVID-19 vaccine acceptance were estimated at the sample mean, overall and by region, for the first approved vaccines [9]. Characteristics of the first approved vaccines differed on efficacy (Pfizer\&BioNTech or Moderna: 95\% vs. AstraZeneca: 60\%) [9,31-33], safety (Pfizer\&BioNTech or Moderna: $1 / 1,000,000$ vs. AstraZeneca: 1/100,000) [9,34], country of the vaccine manufacturer (Pfizer or Moderna: USA vs. BioNTech or AstraZeneca: EU) [9], and place of administration in the initial setting of the mass vaccination campaign in France (Pfizer\&BioNTech or Moderna: mass vaccination center vs. AstraZeneca: general practitioner practice or local pharmacy). Other vaccination characteristics were controlled for at the same level (low duration of vaccine immunity with a booster needed every 6 months; vaccination recommended by the general practitioner; and no information provided about the collective benefits of herd immunity in the initial setting of the mass vaccination campaign in France). 
All analyses were based on two-sided $p$-values, with $p<0.05$ considered to indicate statistical significance. The single hurdle repeated discrete choice model was estimated using maximum likelihood techniques with R statistical software [24].

\section{Results}

\subsection{Prevalence of Detected SARS-CoV-2 Infection in the French Working-Age Population}

A representative sample of 6007 adults aged 18-64 years residing in twelve regions of France participated in the online survey from 30 November to 16 December 2020. Of them, $8.1 \%$ (95\% CI, 7.5-8.8) reported a prior SARS-CoV-2 infection (1.1\% hospital admission for COVID-19; 1.7\% medical diagnosis of COVID-19 without a virology test; and 5.3\% positive test for SARS-CoV-2 infection diagnosis with $(2.9 \%)$ or without $(2.4 \%)$ symptoms). The prevalence rate of detected SARS-CoV-2 infection significantly varied across regions $(p<0.0001)$ according to an East-West gradient (Figure 3; Supplementary Table S2).

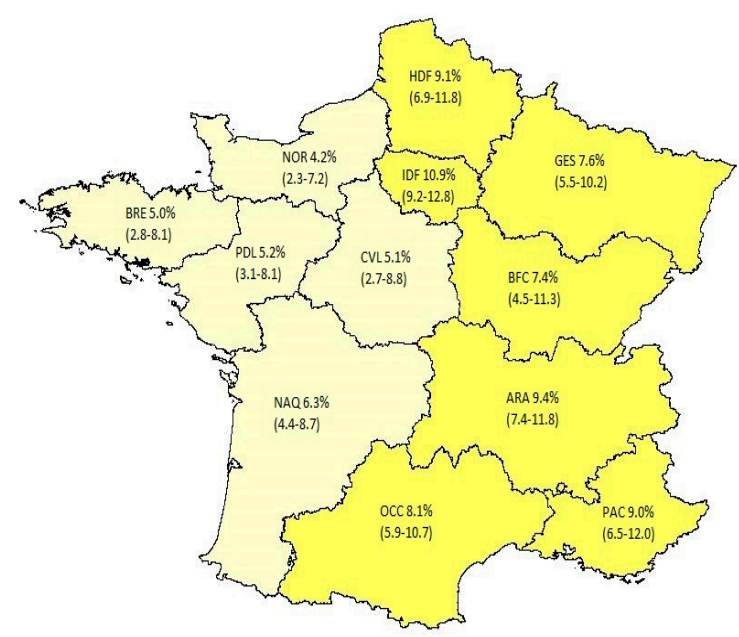

Figure 3. Prevalence of detected SARS-CoV-2 infection in the French working-age population (December 2020). Figure legend: Auvergne-Rhône-Alpes (ARA), Bourgogne-Franche-Comté (BFC), Bretagne (BRE), Cen-tre-Val de Loire (CVL), Grand Est (GES), Hauts-de-France (HDF), Ile-de-France (IDF), Normandie (NOR), Nouvelle-Aquitaine (NAQ), Occitanie (OCC), Pays-de-la-Loire (PDL), and Provences-Alpes-Côte-d'Azur (PAC).

\subsection{COVID-19 Vaccine Hesitancy in Participants without Prior SARS-CoV-2 Infection}

A total of $4806(80 \%)$ participants were randomly allocated to the new survey experiment assessing COVID-19 vaccine hesitancy depending on five vaccine characteristics (Figure 1). Of them, 4415 (91.9\%) did not report prior SARS-CoV-2 infection and were selected for the analysis of COVID-19 vaccine hesitancy. As expected from differences between regional populations, sociodemographic characteristics of the selected participants significantly varied across regions (age: $p<0.05$; education: $p<0.001$; and area of residence: $p<0.001$ ) (Supplementary Table S3). In addition, participants residing in regions with higher prevalence rates of detected SARS-CoV-2 infection more frequently reported COVID-19 experience (having had COVID-19 symptoms without medical confirmation: $p<0.001$; and knowing someone who got COVID-19: $p<0.001)$.

A total of $1823(41.3 \%$ (95\% CI, 39.8-42.8)) participants were identified as refusing COVID-19 vaccination outright. Outright vaccination refusal was not associated with the region of residency in univariate (overall test: $p=0.18$; Supplementary Table S4) and multivariate analyses (overall test: $p=0.60$; Table 1). By contrast, it was independently associated with female gender $(p<0.0001)$, age with an inverted U-shaped relationship $(p<0.0001)$, lower educational achievement $(p<0.01)$, poorer compliance with recommended vaccinations in the past $(p<0.0001)$, no vaccination against 2020/21 influenza $(p<0.0001)$, and lower perceived severity of COVID-19 if infected $(p<0.0001)$. 
Table 1. Behavioral model of COVID-19 vaccination in the French working-age population without prior SARS-CoV-2 infection in December $2020(n=4415)$.

\begin{tabular}{|c|c|c|c|c|}
\hline \multirow[b]{2}{*}{ Determinants of COVID-19 Vaccination } & \multicolumn{2}{|c|}{ Outright Refusal of COVID-19 Vaccination (41.3\%) } & \multicolumn{2}{|c|}{ COVID-19 Vaccine Hesitancy (58.7\%) } \\
\hline & OR $(95 \% \mathrm{CI})$ & $p$-Value & OR $(95 \% \mathrm{CI})$ & $p$-Value \\
\hline \multicolumn{5}{|l|}{ French region of residency (ordered by detected SARS-CoV-2 prevalence) } \\
\hline Normandie (NOR) (ref. Ile-de-France) & $0.92(0.65-1.31)$ & 0.66 & $0.92(0.8-1.10)$ & 0.36 \\
\hline Centre-Val de Loire (CVL) (ref. Ile-de-France) & $0.81(0.53-1.23)$ & 0.32 & $1.09(0.89-1.39)$ & 0.42 \\
\hline Pays-de-la-Loire (PDL) (ref. Ile-de-France) & $0.73(0.49-1.06)$ & 0.10 & $1.12(0.93-1.41)$ & 0.21 \\
\hline Nouvelle-Aquitaine (NAQ) (ref. Ile-de-France) & $1.05(0.74-1.49)$ & 0.79 & $1.02(0.86-1.23)$ & 0.85 \\
\hline Bourgogne-Franche-Comté (BFC) (ref. Ile-de-France) & $0.92(0.61-1.38)$ & 0.69 & $1.22(1.00-1.61)$ & 0.041 \\
\hline Grand Est (GES) (ref. Ile-de-France) & $0.88(0.63-1.23)$ & 0.47 & $0.97(0.84-1.16)$ & 0.75 \\
\hline Provence-Alpes-Côte-d'Azur (PAC) (ref. Ile-de-France) & $0.96(0.70-1.32)$ & 0.82 & $1.02(0.89-1.21)$ & 0.76 \\
\hline Hauts-de-France (HDF) (ref. Ile-de-France) & $0.97(0.73-1.30)$ & 0.85 & $0.99(0.87-1.15)$ & 0.91 \\
\hline Auvergne-Rhône-Alpes (ARA) (ref. Ile-de-France) & $1.05(0.81-1.36)$ & 0.72 & $1.01(0.90-1.16)$ & 0.88 \\
\hline \multicolumn{5}{|l|}{ Vaccine characteristics } \\
\hline Vaccine efficacy of $80 \%$ (ref. $50 \%$ ) & \multirow{10}{*}{\multicolumn{2}{|c|}{ Not applicable }} & $0.58(0.56-0.60)$ & $<0.0001$ \\
\hline Vaccine efficacy of $90 \%$ (ref. 50\%) & & & $0.44(0.43-0.45)$ & $<0.0001$ \\
\hline Vaccine efficacy of $100 \%$ (ref. $50 \%$ ) & & & $0.33(0.32-0.34)$ & $<0.0001$ \\
\hline Vaccine booster every year (ref. booster every 6 months) & & & $0.95(0.90-0.99)$ & 0.029 \\
\hline No vaccine booster needed (ref. booster every 6 months) & & & $0.78(0.75-0.81)$ & $<0.0001$ \\
\hline Unknown risk of serious side effects (ref. $1 / 10,000$ ) & & & $1.54(1.40-1.71)$ & $<0.0001$ \\
\hline Vaccine made in USA (ref. European Union) & & & $1.59(1.44-1.77)$ & $<0.0001$ \\
\hline Vaccine made in China (ref. European Union) & & & $2.40(2.13-2.74)$ & $<0.0001$ \\
\hline Vaccination at GP practice (ref. mass vaccination center) & & & $0.98(0.93-1.03)$ & 0.42 \\
\hline Vaccination at local pharmacy (ref. mass vaccination center) & & & $0.98(0.93-1.03)$ & 0.37 \\
\hline \multicolumn{5}{|l|}{ Background information on COVID-19 vaccination } \\
\hline$>67 \%$ of adults aged $18-64$ years old must be immunized to reach herd immunity (ref. no information) & $0.89(0.76-1.04)$ & 0.16 & $0.92(0.86-0.99)$ & 0.029 \\
\hline$>50 \%$ of adults aged $18-64$ years old must be immunized to reach herd immunity (ref. no information) & $0.99(0.84-1.16)$ & 0.88 & $1.05(0.97-1.14)$ & 0.22 \\
\hline General practitioner recommends vaccination (ref. no opinion) & $1.01(0.88-1.15)$ & 0.90 & $1.02(0.96-1.09)$ & 0.52 \\
\hline \multicolumn{5}{|l|}{ Survey stratification variables (by region of residency) } \\
\hline Men (ref. women) & $0.55(0.48-0.64)$ & $<0.0001$ & $0.71(0.67-0.74)$ & $<0.0001$ \\
\hline 25-34 years (ref. 18-24) & $1.60(1.21-2.12)$ & 0.001 & $1.02(0.90-1.18)$ & 0.81 \\
\hline $35-44$ years (ref. 18-24) & $1.70(1.28-2.26)$ & 0.0003 & $1.04(0.91-1.20)$ & 0.61 \\
\hline $45-54$ years (ref. $18-24$ ) & $1.69(1.28-2.24)$ & 0.0002 & $0.89(0.79-1.00)$ & 0.08 \\
\hline 55-64 years (ref. $18-24$ ) & $1.48(1.11-1.99)$ & 0.008 & $0.86(0.77-0.98)$ & 0.04 \\
\hline High school graduate (ref. some high school) & $0.89(0.75-1.06)$ & 0.20 & $0.86(0.80-0.93)$ & 0.001 \\
\hline University graduate (ref. some high school) & $0.75(0.62-0.90)$ & 0.002 & $0.88(0.82-0.96)$ & 0.007 \\
\hline Number of adults in the household & $0.98(0.88-1.09)$ & 0.74 & $1.11(1.05-1.18)$ & $<0.0001$ \\
\hline Number of children in the household & $1.02(0.94-1.11)$ & 0.57 & $1.01(0.98-1.06)$ & 0.48 \\
\hline Urban area $<100,000$ inhabitants (ref. rural area) & $0.94(0.78-1.13)$ & 0.53 & $1.00(0.92-1.10)$ & 0.97 \\
\hline Urban area $>100,000$ inhabitants (ref. rural area) & $0.78(0.64-0.94)$ & 0.010 & $0.98(0.90-1.08)$ & 0.66 \\
\hline
\end{tabular}


Table 1. Cont.

\begin{tabular}{|c|c|c|c|c|}
\hline \multirow[b]{2}{*}{ Determinants of COVID-19 Vaccination } & \multicolumn{2}{|c|}{ Outright Refusal of COVID-19 Vaccination (41.3\%) } & \multicolumn{2}{|c|}{ COVID-19 Vaccine Hesitancy $(58.7 \%)$} \\
\hline & OR $(95 \% \mathrm{CI})$ & $p$-Value & OR $(95 \% \mathrm{CI})$ & $p$-Value \\
\hline \multicolumn{5}{|l|}{ Variables related to vaccination behavior } \\
\hline Healthcare worker (ref. not working) & $1.01(0.78-1.31)$ & 0.93 & $1.25(1.08-1.49)$ & 0.0005 \\
\hline Worker in contact with the public (ref. not working) & $0.95(0.78-1.14)$ & 0.56 & $1.14(1.03-1.27)$ & 0.005 \\
\hline Worker not in contact with the public (ref. not working) & $1.08(0.89-1.3)$ & 0.45 & $1.14(1.03-1.27)$ & 0.009 \\
\hline Unemployed or furloughed because of the COVID-19 health crisis (ref. not reported) & $0.97(0.81-1.16)$ & 0.76 & $0.95(0.87-1.03)$ & 0.20 \\
\hline Sometimes compliant with recommended vaccination in the past (ref. always) & $2.15(1.86-2.48)$ & $<0.0001$ & $1.61(1.45-1.82)$ & $<0.0001$ \\
\hline Never compliant with recommended vaccination in the past (ref. always) & $5.21(4.22-6.44)$ & $<0.0001$ & $2.92(2.16-4.51)$ & $<0.0001$ \\
\hline Vaccination against influenza 2020/2021 (ref. not reported) & $0.54(0.42-0.67)$ & $<0.0001$ & $0.61(0.58-0.65)$ & $<0.0001$ \\
\hline Pregnancy (ref. not reported) & $1.46(0.73-2.97)$ & 0.28 & $1.37(0.91-2.82)$ & 0.10 \\
\hline Current smoker (ref. never smoker) & $0.93(0.79-1.10)$ & 0.40 & $1.04(0.95-1.13)$ & 0.41 \\
\hline Former smoker (ref. never smoker) & $0.96(0.82-1.12)$ & 0.62 & $1.13(1.04-1.24)$ & 0.002 \\
\hline Obese (ref. normal weight) & $1.04(0.86-1.26)$ & 0.68 & $1.05(0.96-1.16)$ & 0.29 \\
\hline Overweight (ref. normal weight) & $1.15(0.99-1.34)$ & 0.07 & $1.14(1.05-1.24)$ & 0.0006 \\
\hline Hypertension (ref. not reported) & $0.77(0.59-1.00)$ & 0.050 & $0.84(0.76-0.93)$ & 0.004 \\
\hline Diabetes (ref. not reported) & $0.80(0.55-1.15)$ & 0.23 & $1.09(0.93-1.32)$ & 0.31 \\
\hline Other chronic condition (ref. not reported) & $0.99(0.76-1.28)$ & 0.92 & $1.09(0.96-1.25)$ & 0.17 \\
\hline Had COVID-19 symptoms (ref. not reported) & $0.95(0.79-1.13)$ & 0.54 & $1.10(1.00-1.21)$ & 0.031 \\
\hline Had a test for SARS-CoV-2 infection diagnosis (ref. not reported) & $1.01(0.84-1.20)$ & 0.94 & $0.83(0.78-0.90)$ & $<0.0001$ \\
\hline Knows someone who got COVID-19 with hospital admission (ref. not reported) & $0.81(0.67-0.98)$ & 0.032 & $1.12(1.01-1.25)$ & 0.018 \\
\hline Knows someone who got COVID-19 without hospital admission (ref. not reported) & $0.9(0.77-1.04)$ & 0.15 & $1.07(1.00-1.17)$ & 0.06 \\
\hline Curfew in the municipality after 28 October 2020 (ref. after 15 December 2020) & $0.94(0.78-1.14)$ & 0.53 & $0.87(0.81-0.95)$ & 0.005 \\
\hline \multicolumn{5}{|l|}{ Perceived severity of COVID-19 if infected } \\
\hline Very severe (ref. Not severe at all) & $0.46(0.32-0.65)$ & $<0.0001$ & $0.72(0.64-0.82)$ & 0.0001 \\
\hline Somewhat severe (ref. Not severe at all) & $0.55(0.43-0.71)$ & $<0.0001$ & $0.65(0.60-0.71)$ & $<0.0001$ \\
\hline Not particularly severe (ref. Not severe at all) & $0.74(0.58-0.94)$ & 0.015 & $0.75(0.69-0.83)$ & $<0.0001$ \\
\hline Don't know (ref. Not severe at all) & $0.82(0.63-1.08)$ & 0.16 & $1.09(0.94-1.28)$ & 0.24 \\
\hline
\end{tabular}


Among 2592 (58.7\%) participants who did not refuse vaccination outright, vaccine hesitancy was associated with the region of residency (overall test: $p<0.01$; Table 1 ) due to two outlier regions with higher vaccine hesitancy: Bourgogne-Franche-Comté (BFC) $(p<0.05)$ and Occitanie (OCC) $(p<0.01)$. By contrast, vaccine hesitancy was primarily decreased with higher vaccine efficacy, lower risk of serious side-effects, vaccines made in EU rather than USA (or China), and, to a lesser extent, longer duration of vaccine immunity (all $p<0.0001$ ). In addition, sociodemographic and attitudinal factors associated with outright vaccination refusal were also strongly associated with vaccine hesitancy, albeit strengths of association were generally weaker with vaccine hesitancy.

\subsection{COVID-19 Vaccine Acceptance Predicted in December 2020}

In the initial setting of the mass vaccination campaign in France, the behavioral model predicted that outright refusal of COVID-19 vaccination would be present in $42.1 \%$ (95\% CI, 41.6-42.7) of the French working-age population without prior SARS-CoV-2 infection (Figure 4). According to the characteristics of the first approved vaccines, COVID-19 vaccine acceptance would reach at best $43.6 \%(95 \%$ CI, 43.0-44.1) for a BioNTech vaccine promoted as made in EU rather than USA (Figure 4) and would be lower for Pfizer or Moderna vaccines (38.4\% (95\% CI, 37.8-38.9)); Supplementary Figure S1) and the AstraZeneca vaccine (31.1\% (95\% CI, 30.6-31.6)); Supplementary Figure S2).

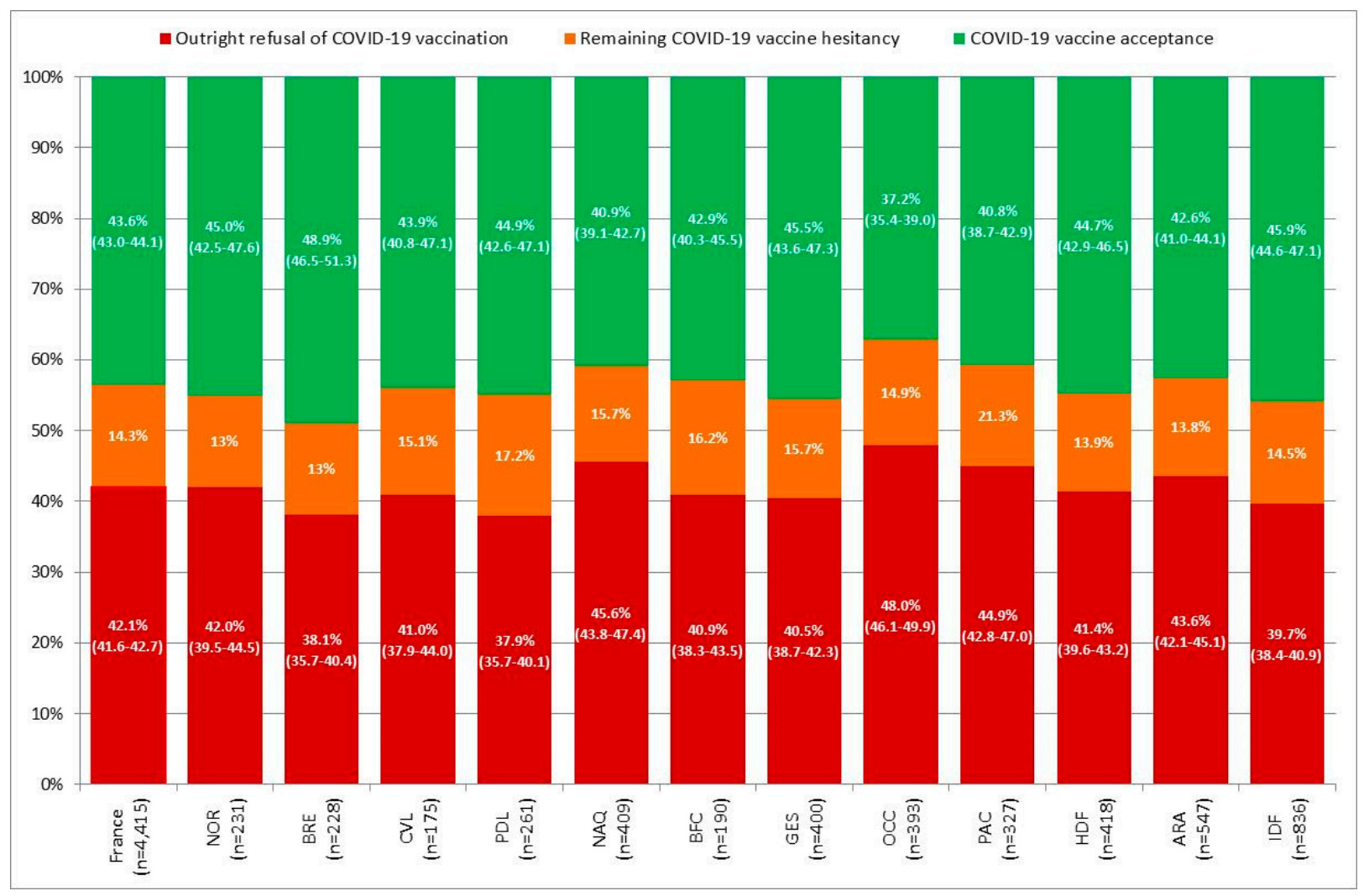

Figure 4. Acceptance of BioNTech vaccine predicted in the French working-age population, overall and by region (December 2020). Figure legend: Auvergne-Rhône-Alpes (ARA), Bourgogne-Franche-Comté (BFC), Bretagne (BRE), Cen-tre-Val de Loire (CVL), Grand Est (GES), Hauts-de-France (HDF), Ile-de-France (IDF), Normandie (NOR), Nouvelle-Aquitaine (NAQ), Occitanie (OCC), Pays-de-la-Loire (PDL), and Provences-Alpes-Côte-d'Azur (PAC). French regions of residency are ordered by detected SARS-CoV-2 prevalence.

Acceptance of a BioNTech vaccine was estimated at the sample mean of each region of residency. Figure 4 presents regional estimates ordered by increasing prevalence of detected SARS-CoV-2 infection. Outright refusal of COVID-19 vaccination would significantly decrease from $48.0 \%$ (95\% CI, 46.1-49.9) in Occitanie (OCC) to $37.9 \%(95 \%$ CI, 35.7-40.1) 
in Pays-de-la-Loire (PDL), although it was not correlated with the prevalence of detected SARS-CoV-2 infection ( $\rho=0.27 ; p=0.40)$. Similarly, acceptance of a BioNTech vaccine would significantly increase from $37.2 \%$ (95\% CI, 35.4-39.0) in Occitanie (OCC) to $48.9 \%$ (95\% CI, 46.5-51.3) in Bretagne (BRE), although it was not correlated with the prevalence of detected SARS-CoV-2 infection $(\rho=-0.26 ; p=0.42)$.

\section{Discussion}

In this large and representative survey of 6007 adults aged 18-64 years and residing in France, 8.1\% (95\% CI, 7.5-8.8) reported a prior SARS-CoV-2 infection in December 2020, with regional variations according to an East-West gradient $(p<0.0001)$. In participants without prior SARS-CoV-2 infection, COVID-19 vaccine hesitancy was substantial, including $41.3 \%$ (95\% CI, 39.8-42.8) outright refusal of COVID-19 vaccination. Taking into account five characteristics of the first approved vaccines (efficacy, duration of immunity, safety, country of the vaccine manufacturer, and place of administration) as well as the initial setting of the mass vaccination campaign in France, COVID-19 vaccine acceptance would reach $43.6 \%$ (95\% CI, 43.0-44.1) at best among working-age adults without prior SARS-CoV2 infection. COVID-19 vaccine acceptance was primarily driven by vaccine characteristics, sociodemographic and attitudinal factors. Considering the region of residency as a proxy of the likelihood of getting infected, our study findings do not support the assumption that SARS-CoV-2 infection risk is associated with COVID-19 vaccine acceptance.

To our knowledge, this is the first study assessing the association between SARS-CoV-2 infection risk and COVID-19 vaccination intentions. France provides a natural experiment to assess this association as SARS-CoV-2 pandemic initially spread in the North-East and Ile-de-France regions and was controlled by a nationwide lockdown ordered on 17 March 2020 [2]. Accordingly, the heterogeneity of SARS-CoV-2 infection rates across regions was high after the first wave (11 May 2020) and only marginally reduced during the second wave (31 October 2020) [22]. In our study, 8.1\% (95\% CI, 7.5-8.8) of participants reported a prior SARS-CoV-2 infection during the second wave of the SARS-CoV-2 pandemic in December 2020. In comparison, the detection rate of SARS-CoV-2 infection in the French working-age population was similarly estimated at 7.8\% (95\% CI, 6.0-10.0) as of 15 January 2021 in an epidemiological modeling study [22]. In addition, the external validity of our study was enhanced by the significant correlation of regional estimates between the two studies $(\rho=0.81 ; p<0.01)$ (Supplementary Table S2).

We assessed COVID-19 vaccination intentions in participants without prior SARS-CoV2 infection with use of a two-part behavioral model [24]. As compared to opinion surveys asking about vaccination intentions for vaccines with unspecified characteristics [16-18], it allowed the identification of participants who would never choose to be vaccinated regardless of five vaccine characteristics (outright refusal of vaccination) and those who are hesitant and whose choice to be vaccinated would depend on vaccine characteristics [9]. In participants who did not refuse vaccination outright, we found that vaccine efficacy, vaccine safety, and the country of the vaccine manufacturer were primary drivers of COVID-19 vaccine acceptance as in previous discrete choice experiments [24,25,35-38]. In addition, participants were randomly allocated to two discrete choice experiment designs presenting five $(80 \%)$ vs. four $(20 \%)$ vaccine characteristics (Figure 1), and we found robust estimates across the two survey experiments regarding outright refusal of vaccination $(41.3 \%$ (95\% CI, 39.8-42.8)) vs. $42.5 \%$ (95\% CI, 41.1-43.8), respectively) and overall acceptance of the best (BioNTech) vaccine (43.6\% (95\% CI, 43.0-44.1) vs. 44.0\% (95\% CI, 42.7-45.2), respectively) [25].

Our study findings do not support the assumption that SARS-CoV-2 infection risk is associated with COVID-19 vaccination intentions in the working-age population. At the individual level, the region of residency was not an independent predictor of outright vaccination refusal. Two regions (Bourgogne-Franche-Comté and Occitanie) were independently associated with higher vaccine hesitancy as compared to other regions. However, they were in the middle range rather than the ends of prevalence rates of detected SARS-CoV-2 
infection (Supplementary Table S2). At the macro level, COVID-19 vaccine acceptance significantly varied across regions, although it was uncorrelated with prevalence rates of detected SARS-CoV-2 infection $(\rho=-0.26 ; p=0.42)$. By contrast, regional variations in COVID-19 vaccine acceptance were primarily explained by differences on sociodemographic characteristics (age, education, and area of residency) that were in turn consistently associated with COVID-19 vaccine hesitancy $[24,25]$.

The main limitation of our study relates to its timing. COVID-19 vaccination intentions may continuously evolve in relation to the dynamic of the SARS-CoV-2 pandemic and associated non-pharmaceutical interventions [2,3] as well as information on COVID-19 vaccine uptake made available to the public in almost real-time [39]. Nevertheless, our study findings support that COVID-19 vaccine hesitancy was profoundly anchored in multiple, large sociodemographic strata of the working-age population (women, 25-54 years old, lower educational achievement, rural areas) before mass vaccination started in the elderly in France.

Another limitation is that sample size was limited in regions that are sparsely populated, with a minimum of 234 (3.9\%) participants in the Centre-Val de Loire (CVL) region. However, the study was conducted among the working-age population who is at high risk of SARS-CoV-2 infection as compared to the elderly $[22,23]$. To avoid spurious association, we excluded participants reporting prior SARS-CoV-2 infection from the analysis of COVID-19 vaccine hesitancy as they were excluded from vaccine phase 3 trials [31-33], and vaccination was not recommended for them in the initial setting of the mass vaccination campaign in France [40]. In addition, our behavioral model of COVID-19 vaccine hesitancy was controlled for a large set of independent factors including COVID-19 experience and the perceived severity of COVID-19 that may confound the association between the likelihood of getting infected and COVID-19 vaccination intentions [19-21].

Finally, Corsica was not included in our study as this region was underrepresented in the BVA online survey research panel. Corsica accounts for $0.5 \%$ of the working-age population in France (2020 national census data), and its inclusion may have biased our correlation estimate between the prevalence of detected SARS-CoV-2 infection and COVID19 vaccination hesitancy at the regional level. In addition, Corsica was associated with one of the lowest detection rates of SARS-CoV-2 infection as of 15 January 2021 (4.0\% (95\% CI, 3.1-5.3) of the working-age population) [22] but remained in the middle range of COVID-19 vaccination coverage recorded by region (e.g., $42.2 \%$ as compared to $43.4 \%$ (min: $40.0 \%$; max: $47.0 \%$ ) of the working-age population in other regions of metropolitan France as of 11 July 2021) [41], further supporting that there is no association between SARS-CoV-2 infection risk and COVID-19 vaccination in the working-age population.

In summary, our survey experiment supports that COVID-19 vaccine hesitancy was deeply anchored in the French working-age population in December 2020 and primarily driven by vaccine characteristics, sociodemographic and attitudinal factors, but unrelated to SARS-CoV-2 infection risk at the rather low levels estimated in 2020. Accordingly, French regions that were initially spared during the first wave of SARS-CoV-2 pandemic may remain at high risk of local outbreaks of SARS-CoV-2 pandemic despite availability of COVID-19 vaccination services [10-12].

Supplementary Materials: The following are available online at https: / www.mdpi.com/article / 10.3390/vaccines9111364/s1, Table S1: Prevalence of detected SARS-CoV-2 infection in the French working age population, Table S2: Characteristics of participants without prior SARS-CoV-2 infection by region of residency (December 2020, France), Table S3: Outright refusal of COVID-19 vaccination (December 2020, France), Table S4. Outright refusal of COVID-19 vaccination (December 2020, France), Figure S1: Acceptance of Pfizer or Moderna vaccines predicted in the working age population (December 2020, France), Figure S2: Acceptance of AstraZeneca vaccine predicted in the working age population (December 2020, France). 
Author Contributions: Conceptualization, M.S.; methodology, M.S., V.W. and S.L.; software (NGENE software, ChoiceMetrics, 2014), V.W.; validation, F.V., V.W., P.A., F.A., S.L. and M.S.; formal analysis, S.L.; resources, M.S.; data curation, M.S. and S.L.; writing-original draft preparation, F.V. and M.S.; writing-review and editing, F.V., V.W., P.A., F.A., S.L. and M.S.; project administration, M.S.; funding acquisition, M.S. All authors have read and agreed to the published version of the manuscript.

Funding: Online survey experiments were funded by Santé publique France. This research was funded by Agence Nationale de la Recherche (ANR-21-CO16-0006-01).

Institutional Review Board Statement: The approval of an ethics committee was not required for this non-interventional research study (Act L1121 of French Public Health Code). The data management of the BVA online survey research panel preserves the anonymity of participants and is approved by the French National Commission for Data Protection (CNIL) in accordance with the General Data Protection Regulation of the European Union (approval renewal on 25 May 2018).

Informed Consent Statement: Informed consent was obtained from all subjects involved in the study.

Data Availability Statement: De-identified cross-sectional data and R program used in this study can be made available on reasonable request from the corresponding author.

Acknowledgments: The Department of methodology and innovation in prevention (SSMIP) of the teaching hospital of Bordeaux is partially funded by Agence Régionale de Santé (ARS) NouvelleAquitaine, France. Health Economics Research Unit (HERU) is funded by the Chief Scientist Office (CSO) of the Scottish Government Health and Social Care Directorates. Centre de Calcul Intensif d'Aix-Marseille is acknowledged for granting access to its high performance computing resources. The CoVaMax Study Group includes: François Alla, Angela Baron, Coline Bourreau, Léo Donzel Godinot, Michaël Schwarzinger, Fanny Velardo (Department of methodology and innovation in prevention, Bordeaux university hospital, 33000 Bordeaux, France); Marcela Benavides, Linda Cambon, Laurence Kotobi, Elodie Richard (University of Bordeaux, Inserm UMR 1219-Bordeaux Population Health, 33000 Bordeaux, France); Pierre Arwidson, Isabelle Bonmarin, Stéphanie Vandentorren (Santé Publique France, 94410 Saint Maurice, France); Stéphane Luchini (Aix-Marseille Univ, CNRS, EHESS, Centrale Marseille, AMSE, 13001 Marseille, France); Verity Watson (Health Economics Research Unit (HERU), University of Aberdeen, Aberdeen AB25 2ZD, UK).

Conflicts of Interest: The authors declare no conflict of interest. The funders had no role in the design of the study; in the collection, analyses, or interpretation of data; in the writing of the manuscript, or in the decision to publish the results.

\section{References}

1. World Health Organization. WHO Director-General's Opening Remarks at the Media Briefing on COVID-19. 11 March 2020; WHO: Geneva, Switzerland, 2020.

2. European Centre for Disease Prevention and Control. Weekly COVID-19 Country Overview. 2021. Available online: https: / / www.ecdc.europa.eu/en/covid-19/country-overviews (accessed on 3 November 2021).

3. Bendavid, E.; Oh, C.; Bhattacharya, J.; Ioannidis, J.P.A. Assessing mandatory stay-at-home and business closure effects on the spread of COVID-19. Eur. J. Clin. Investig. 2021, 51, e13484. [CrossRef]

4. Islam, N.; Shkolnikov, V.M.; Acosta, R.J.; Klimkin, I.; Kawachi, I.; Irizarry, R.A.; Alicandro, G.; Khunti, K.; Yates, T.; Jdanov, D.A.; et al. Excess deaths associated with covid-19 pandemic in 2020: Age and sex disaggregated time series analysis in 29 high income countries. BMJ 2021, 373, n1137. [CrossRef] [PubMed]

5. Roser, M.; Ritchie, H.; Ortiz-Ospina, E.; Hasell, J. Coronavirus (COVID-19) Deaths. 2020. Available online: https:/ / ourworldindata. org/covid-deaths (accessed on 3 November 2021).

6. Moderna. Moderna's COVID-19 Vaccine Candidate Meets Its Primary Efficacy Endpoint in the First Interim Analysis of the Phase 3 COVE Study. 16 November 2020. Available online: https:/ /investors.modernatx.com/news-releases/news-release-details/ modernas-covid-19-vaccine-candidate-meets-its-primary-efficacy (accessed on 3 November 2021).

7. Pfizer; BioNTech. Pfizer and BioNTech Conclude Phase 3 Study of COVID-19 Vaccine Candidate, Meeting All Primary Efficacy Endpoints. 18 November 2020. Available online: https:/ /www.pfizer.com/news/press-release/press-release-detail/pfizer-andbiontech-conclude-phase-3-study-covid-19-vaccine (accessed on 3 November 2021).

8. AstraZeneca; Oxford University. AZD1222 Vaccine Met Primary Efficacy Endpoint in Preventing COVID-19. 23 November 2020. Available online: https:/ / www.astrazeneca.com/media-centre/press-releases/2020/azd1222hlr.html (accessed on 3 November 2021). 
9. European Medicines Agency. COVID-19 Vaccines: Authorised for Use in the European Union. 2021. Available online: https://www.ema.europa.eu/en/human-regulatory/overview/public-health-threats/coronavirus-disease-covid-19 / treatments-vaccines / covid-19-vaccines (accessed on 31 May 2021).

10. Saad-Roy, C.M.; Wagner, C.E.; Baker, R.E.; Morris, S.E.; Farrar, J.; Graham, A.L.; Levin, S.A.; Mina, M.J.; Metcalf, C.J.E.; Grenfell, B.T. Immune life history, vaccination, and the dynamics of SARS-CoV-2 over the next 5 years. Science 2020, 370, 811-818. [CrossRef]

11. Olivera Mesa, D.; Hogan, A.B.; Watson, O.J.; Charles, G.D.; Hauck, K.; Ghani, A.C.; Winskill, P. Report 43: Quantifying the Impact of Vaccine Hesitancy in Prolonging the Need for Non-Pharmaceutical Interventions to Control the COVID-19 Pandemic; Imperial College: London, UK, 2021. [CrossRef]

12. Moore, S.; Hill, E.M.; Tildesley, M.J.; Dyson, L.; Keeling, M.J. Vaccination and non-pharmaceutical interventions for COVID-19: A mathematical modelling study. Lancet Infect. Dis. 2021, 21, 793-802. [CrossRef]

13. MacDonald, N.E.; Sage Working Group on Vaccine Hesitancy. Vaccine hesitancy: Definition, scope and determinants. Vaccine 2015, 33, 4161-4164. [CrossRef] [PubMed]

14. Dube, E.; Laberge, C.; Guay, M.; Bramadat, P.; Roy, R.; Bettinger, J. Vaccine hesitancy: An overview. Hum. Vaccines Immunother. 2013, 9, 1763-1773. [CrossRef] [PubMed]

15. de Figueiredo, A.; Simas, C.; Karafillakis, E.; Paterson, P.; Larson, H.J. Mapping global trends in vaccine confidence and investigating barriers to vaccine uptake: A large-scale retrospective temporal modelling study. Lancet 2020, 396, 898-908. [CrossRef]

16. Lin, C.; Tu, P.; Beitsch, L.M. Confidence and Receptivity for COVID-19 Vaccines: A Rapid Systematic Review. Vaccines 2020, 9, 16. [CrossRef]

17. Robinson, E.; Jones, A.; Lesser, I.; Daly, M. International estimates of intended uptake and refusal of COVID-19 vaccines: A rapid systematic review and meta-analysis of large nationally representative samples. Vaccine 2021, 39, 2024-2034. [CrossRef]

18. Sallam, M. COVID-19 Vaccine Hesitancy Worldwide: A Concise Systematic Review of Vaccine Acceptance Rates. Vaccines 2021, 9, 160. [CrossRef]

19. Brewer, N.T.; Chapman, G.B.; Gibbons, F.X.; Gerrard, M.; McCaul, K.D.; Weinstein, N.D. Meta-analysis of the relationship between risk perception and health behavior: The example of vaccination. Health Psychol. 2007, 26, 136-145. [CrossRef]

20. Bish, A.; Yardley, L.; Nicoll, A.; Michie, S. Factors associated with uptake of vaccination against pandemic influenza: A systematic review. Vaccine 2011, 29, 6472-6484. [CrossRef]

21. Caserotti, M.; Girardi, P.; Rubaltelli, E.; Tasso, A.; Lotto, L.; Gavaruzzi, T. Associations of COVID-19 risk perception with vaccine hesitancy over time for Italian residents. Soc. Sci. Med. 2021, 272, 113688. [CrossRef] [PubMed]

22. Hoze, N.; Paireau, J.; Lapidus, N.; Tran Kiem, C.; Salje, H.; Severi, G.; Touvier, M.; Zins, M.; de Lamballerie, X.; Levy-Bruhl, D.; et al. Monitoring the proportion of the population infected by SARS-CoV-2 using age-stratified hospitalisation and serological data: A modelling study. Lancet Public Health 2021, 6, e408-e415. [CrossRef]

23. O'Driscoll, M.; Ribeiro Dos Santos, G.; Wang, L.; Cummings, D.A.T.; Azman, A.S.; Paireau, J.; Fontanet, A.; Cauchemez, S.; Salje, H. Age-specific mortality and immunity patterns of SARS-CoV-2. Nature 2021, 590, 140-145. [CrossRef] [PubMed]

24. Schwarzinger, M.; Watson, V.; Arwidson, P.; Alla, F.; Luchini, S. COVID-19 vaccine hesitancy in a representative working-age population in France: A survey experiment based on vaccine characteristics. Lancet Public Health 2021, 6, e210-e221. [CrossRef]

25. Schwarzinger, M.; Watson, V.; Arwidson, P.; Alla, F.; Luchini, S.; CoVaMax Study Group. Evolution of COVID-19 vaccine hesitancy between June and December 2020: A replication experiment in the French working age population. Vaccines 2021, submitted.

26. Betsch, C.; Böhm, R.; Korn, L.; Holtmann, C. On the benefits of explaining herd immunity in vaccine advocacy. Nat. Hum. Behav. 2017, 1, 0056. [CrossRef]

27. Schwarzinger, M.; Flicoteaux, R.; Cortarenoda, S.; Obadia, Y.; Moatti, J.P. Low acceptability of A/H1N1 pandemic vaccination in French adult population: Did public health policy fuel public dissonance? PLoS ONE 2010, 5, e10199. [CrossRef]

28. Reiter, P.L.; Pennell, M.L.; Katz, M.L. Acceptability of a COVID-19 vaccine among adults in the United States: How many people would get vaccinated? Vaccine 2020, 38, 6500-6507. [CrossRef] [PubMed]

29. Hauber, A.B.; Gonzalez, J.M.; Groothuis-Oudshoorn, C.G.; Prior, T.; Marshall, D.A.; Cunningham, C.; MJ, I.J.; Bridges, J.F. Statistical Methods for the Analysis of Discrete Choice Experiments: A Report of the ISPOR Conjoint Analysis Good Research Practices Task Force. Value Health 2016, 19, 300-315. [CrossRef]

30. von Haefen, R.H.; Massey, D.M.; Adamowicz, W.L. Serial Nonparticipation in Repeated Discrete Choice Models. Am. J. Agric. Econ. 2005, 87, 1061-1076. [CrossRef]

31. Polack, F.P.; Thomas, S.J.; Kitchin, N.; Absalon, J.; Gurtman, A.; Lockhart, S.; Perez, J.L.; Perez Marc, G.; Moreira, E.D.; Zerbini, C.; et al. Safety and Efficacy of the BNT162b2 mRNA COVID-19 Vaccine. N. Engl. J. Med. 2020, 383, 2603-2615. [CrossRef] [PubMed]

32. Baden, L.R.; El Sahly, H.M.; Essink, B.; Kotloff, K.; Frey, S.; Novak, R.; Diemert, D.; Spector, S.A.; Rouphael, N.; Creech, C.B.; et al. Efficacy and Safety of the mRNA-1273 SARS-CoV-2 Vaccine. N. Engl. J. Med. 2020, 385, 1774-1785. [CrossRef] [PubMed]

33. Voysey, M.; Clemens, S.A.C.; Madhi, S.A.; Weckx, L.Y.; Folegatti, P.M.; Aley, P.K.; Angus, B.; Baillie, V.L.; Barnabas, S.L.; Bhorat, Q.E.; et al. Safety and efficacy of the ChAdOx1 nCoV-19 vaccine (AZD1222) against SARS-CoV-2: An interim analysis of four randomised controlled trials in Brazil, South Africa, and the UK. Lancet 2021, 397, 99-111. [CrossRef] 
34. Ostergaard, S.D.; Schmidt, M.; Horvath-Puho, E.; Thomsen, R.W.; Sorensen, H.T. Thromboembolism and the Oxford-AstraZeneca COVID-19 vaccine: Side-effect or coincidence? Lancet 2021, 397, 1441-1443. [CrossRef]

35. Kreps, S.; Prasad, S.; Brownstein, J.S.; Hswen, Y.; Garibaldi, B.T.; Zhang, B.; Kriner, D.L. Factors Associated With US Adults' Likelihood of Accepting COVID-19 Vaccination. JAMA Netw. Open 2020, 3, e2025594. [CrossRef]

36. Craig, B.M. United States COVID-19 Vaccination Preferences (CVP): 2020 Hindsight. Patient 2021, 14, 309-318. [CrossRef]

37. Borriello, A.; Master, D.; Pellegrini, A.; Rose, J.M. Preferences for a COVID-19 vaccine in Australia. Vaccine 2021, 39, 473-479. [CrossRef]

38. McPhedran, R.; Toombs, B. Efficacy or delivery? An online Discrete Choice Experiment to explore preferences for COVID-19 vaccines in the UK. Econ. Lett. 2021, 200, 109747. [CrossRef]

39. Agranov, M.; Elliott, M.; Ortoleva, P. The importance of Social Norms against Strategic Effects: The case of COVID-19 vaccine uptake. Econ. Lett. 2021, 206, 109979. [CrossRef] [PubMed]

40. Haute Autorité de Santé. Décision $n^{\circ}$ 2020.0308/AC/SEESP Recommandations Complétant la Recommandation Vaccinale «Stratégie de Vaccination Contre le SARS-Cov-2-Recommandations Préliminaires sur la Stratégie de Priorisation des Populations à Vacciner» [Recommendations Supplementing the Vaccine Recommendation "Sars-Cov-2 Vaccination Strategy-Preliminary Recommendations on the Strategy for Prioritizing Populations to Be Vaccinated"]. 17 December 2020. Available online: https: / / www.has-sante.fr/jcms /p_3225548/fr/decision-n-2020-0308/ac/seesp-du-17-decembre-2020-du-college-de-lahaute-autorite-de-sante-portant-adoption-de-recommandations-completant-la-recommandation-vaccinale-strategie-devaccination-contre-le-sars-cov-2-recommandations-preliminaires-sur-la-strategie-de-priorisation-des-populations-a-vacciner (accessed on 3 November 2021).

41. Assurance Maladie. Les Données de la Vaccination Contre la COVID-19 [COVID-19 Vaccination Data]. 2021. Available online: https:/ / datavaccin-covid.ameli.fr/pages/home/ (accessed on 3 November 2021). 